\title{
BAMBI promotes macrophage proliferation and differentiation in gliomas
}

\author{
DAN WANG ${ }^{1}$, XIAOLAI CHEN ${ }^{1}$ and RAN ZHANG $^{2}$ \\ ${ }^{1}$ Department of Neurosurgery, Hunan Provincial People's Hospital, The First Affiliated Hospital \\ of Hunan Normal University, Changsha, Hunan 410005; ${ }^{2}$ Department of Immunology, Hunan \\ Normal University School of Medicine, Changsha, Hunan 410031, P.R. China
}

Received November 30, 2015; Accepted November 15, 2016

DOI: $10.3892 / \mathrm{mmr} .2017 .8320$

\begin{abstract}
The present study investigated the capacity of Bone morphogenic protein and activin membrane-bound inhibitor homolog (BAMBI) to regulate the migration and differentiation of macrophages in gliomas. Using a migration assay, it was determined that BAMBI stimulated monocytes migration in a dose-dependent effect. When induced by phorbol myristate acetate (PMA) the monocytes differentiated into macrophages, and BAMBI also increased the migration of PMA-induced macrophages compared with control cells. The expression of CD68 and BAMBI protein and mRNA in glioma and normal specimens were detected using immunohistochemistry and reverse transcription-quantitative polymerase chain reaction, respectively. The localization of BAMBI was primarily in macrophages, as demonstrated by staining for the macrophage marker CD68, and the mRNA expression of CD68 and BAMBI were higher in gliomas compared to normal tissues. In addition, the mRNA expression of CD68 and BAMBI were positively correlated $\left(\mathrm{R}^{2}=0.64\right)$. After treatment with $50 \mathrm{nM}$ PMA and 10 nM BAMBI for 48 h, RAW 264.7 macrophages were exhibited dendrite-like morphology, indicating that the co-treatment promoted the differentiation of monocytes to macrophages. The expression of specific markers of M1 [inducible nitric oxide synthase (iNOS) and interleukin (IL)-12] and M2 (IL-10 and arginase 1) type macrophages was determined following $10 \mathrm{nM}$ BAMBI treatment. BAMBI promoted the expression of M1 markers, whereas the M2 markers were not affected, which indicated that BAMBI induced differentiation of M1 type macrophages. These results indicate that BAMBI may be involved in macrophage differentiation in gliomas.
\end{abstract}

Correspondence to: Professor Xiaolai Chen, Department of Neurosurgery, Hunan Provincial People's Hospital, The First Affiliated Hospital of Hunan Normal University, 61 West Jiefang Road, Changsha, Hunan 410005, P.R. China

E-mail: xiaolai_15chen@sina.com

Key words: BAMBI, macrophage, gliomas, iNOS, IL-12

\section{Introduction}

Inflammation has been previously defined to have important role in various pathologies, including gliomas (1-3). Currently, there is no clear cause of glioma development. The probable causative factors may involve infectious agents, dietary factors or estrogens (3). Within the various inflammatory cell populations of the nervous system, it has been recognized that macrophages are one of the important inflammatory components. However, there are conflicting results regarding the contributory mechanisms of macrophages in the inflammatory process.

Previous studies demonstrated that chronic inflammation may be associated with high-grade gliomas and then result in a high risk of glioma development $(4,5)$. Increased numbers of macrophages have been detected in chronic inflammatory infiltrates of gliomas $(1,6)$. They have also been associated with tissue injuries. Compared with normal tissues and cells, numerous inflammatory cytokines are overexpressed in gliomas $(1,7,8)$. However, how immune infiltrates are involved in the progression and prognosis of gliomas remains unclear. A high level of tumor-associated macrophages (TAMs) is an independent predictor for disease-free survival for ovarian cancer (9). Additionally, a polarized M2 phenotype is produced by tumor-derived and $\mathrm{T}$ cell-derived cytokines in macrophages which may be associated with cancer progression (9). Decreased number of macrophages reduced angiogenesis and tumor growth, and macrophages regulate hormonal resistance in gliomas (10).

Bone morphogenic protein (BMP) and activin membrane-bound inhibitor homolog (BAMBI) is a transmembrane glycoprotein which shares certain homology with-type I receptors of the transforming growth factor- $\beta$ (TGF- $\beta$ ) family (11). The extracellular domain of BAMBI is homologous to the protein sequence of T-box brain protein 1 (TBR1). Thus, BAMBI is a pseudoreceptor of TBR1. BAMBI is widely-expressed, including in nervous system and ovarian cancer $(11,12)$. BAMBI is also overexpressed in different types of cancer $(11,13)$. Upregulation of BAMBI is correlated with tumor growth and metastasis via escape from growth arrest by TGF $\beta$ (14). Furthermore, BAMBI has been demonstrated to be that co-expressed with BMP, TGF- $\beta$ and activin, which indicates a potential function of BAMBI in mediating 
macrophage proliferation (15). In addition, the expression of BAMBI is regulated by lysosomal/autolysosomal degradation indicating that there is an association between BAMBI and immune activities in renal endothelial cells (16). In addition, these effects of BAMBI may be crucial in cancer progression via stimulating macrophage proliferation (16). However, the link between immunomodulatory activity of BAMBI and inflammation-associated glioma development requires further investigation.

It has been previously demonstrated that macrophages have an important role in cancer (17). BAMBI has been demonstrated to be highly expressed in macrophages (18). However, few studies have investigated the direct effect of BAMBI on monocyte/macrophage migration and differentiation, or the association between BAMBI expression levels and macrophage density human glioma in specimens. In the present study, the effect of BAMBI on monocytes and macrophages was evaluated via cell migration assay, reverse transcription-quantitative polymerase chain reaction (RT-qPCR) and western blotting, and immunohistochemistry using gliomas tissues. The results demonstrated that BAMBI increased monocyte migration and activated macrophages in vitro, and that the BAMBI expression levels were positively associated with macrophage density in human glioma.

\section{Materials and methods}

Patients. Formalin-fixed, paraffin-embedded adjacent normal tissue $(n=27)$ and glioma $(n=27$; Gleason score, 6-7) specimens were obtained from Hunan Provincial People's Hospital of Hunan Normal University (Changsha, China). The characteristics of the patients are presented in Table I. The specimens were diagnosed and scored by specialized pathologists via hematoxylin and eosin staining (Beyotime Institute of Biotechnology, Haimen, China). Written informed consent was obtained from each patient and the study was approved by the Ethics Committees of Hunan Provincial People's Hospital of Hunan Normal University.

Cell lines. RAW 264.7 macrophages were purchased from American Type Culture Collection (ATCC; Manassas, VA, USA) and the cells were cultivated in Dulbecco's modified Eagle's medium (DMEM) (Gibco; Thermo Fisher Scientific, Inc., Waltham, MA, USA) supplemented with $10 \%$ fetal bovine serum (FBS) (Gibco; Thermo Fisher Scientific, Inc.). THP-1 monocytes (ATCC) were cultured in RPMI-1640 (Gibco; Thermo Fisher Scientific, Inc.) with 10\% FBS. Differentiation of monocytes into macrophages was induced using $50 \mathrm{nM}$ phorbol myristate acetate (PMA; Sigma-Aldrich; Merck Millipore, Darmstadt, Germany) for 48 h (11). The U-87 glioma cell line (ATCC) was cultivated in RPMI-1640 medium with $10 \%$ FBS. All the cells were cultured at $37^{\circ} \mathrm{C}$ with $5 \% \mathrm{CO}_{2}$.

Cell migration assay. The cell migration was assayed using 48-well Boyden chambers (Neuro Probe, Inc., Gaithersburg, MD, USA). Monocytes $\left(2 \times 10^{6}\right.$ cell $\left./ \mathrm{ml}\right)$ were seeded on the top of the chamber membrane. RPMI-1640 containing 10\% FBS (Gibco-BRL, Grand Island, NY, USA) and 1 or $10 \mathrm{nM}$ BAMBI (Sino Biological, Inc., Beijing, China) was added to the bottom of the well. RPMI-1640 without FBS was added to the top
Table I. Clinicopathological characteristics of the patients within the study.

Characteristic

Sex

Male 13

Female

Age (years)

20-45 16

46-60

Glioma histopathology grade

I-II

III-IV

chamber. Control wells received no treatment. After incubation for $10 \mathrm{~h}$, the migrated cells were stained using Crystal Violet Staining Solution for $15 \mathrm{~min}$ at $37^{\circ} \mathrm{C}$ (Sangon Biotech Co., Ltd., Shanghai, China). The migrated cells were imaged using a Leica 400 light microscope (Leica Microsystems, Inc., Buffalo Grove, IL, USA) and the number of cells in 10 fields was calculated by Image-Pro Plus software, version 5.0 (Media Cybernetics, Inc., Rockville, MD, USA).

$R T$ - $q P C R$. RNA was extracted from cells or tissues using RNeasy Mini kit (Qiagen, Inc., Valencia, CA, USA). Total RNA $(1 \mu \mathrm{g})$ was reverse transcribed to cDNA using the Verso cDNA Synthesis kit (Thermo Fisher Scientific, Inc.), according to the manufacturer's instructions. The qPCR reaction was performed on an ABI 7500 Real-Time PCR system (Applied Biosystems; Thermo Fisher Scientific, Inc.) using Premix Ex Taq $^{\text {TM }}$ PCR kit (Perfect Real-Time; Takara Biotechnology Co., Ltd., Dalian, China) with the following conditions: Initial denaturating for $30 \mathrm{sec}$ at $95^{\circ} \mathrm{C}$; then 40 cycles of $30 \mathrm{sec}$ at $95^{\circ} \mathrm{C}$ and $30 \mathrm{sec}$ at $60^{\circ} \mathrm{C}$. The primers used were as follows: BAMBI forward, 5'-CTCAAATTCCCCACTCACCCA-3' and reverse, 5'-GCTGATACCTGTTTCCTTGTCCTG-3'; CD68 forward, 5'-CACGCAGCACAGTGGACATTC-3' and reverse, 5'-GCCTGGAGCCTCAGGGAGA-3'; and GAPDH forward, 5'-AATCCCATCACCATCTTCCA-3' and reverse, 5'-TGG ACTCCACGACGTACTCA-3'. The relative expression levels were calculated using the $2^{-\Delta \Delta C q}$ method (19). Each sample was assayed in triplicate.

Western blotting. Protein expression was detected by western blot analysis. Cells were lysed by radioimmunoprecipitation buffer (Beyotime Institute of Biotechnology). The protein concentration in each sample was determined using a Bradford kit (Beyotime Institute of Biotechnology), $20 \mu \mathrm{g}$ total protein was separated by $10 \%$ SDS-PAGE (Beyotime Institute of Biotechnology). The proteins were transferred onto a polyvinylidene fluoride membrane (EMD Millipore, Billerica, MA, USA). The membrane was blocked with $4 \%$ skimmed milk at room temperature for $1 \mathrm{~h}$, and subsequently primary antibodies were incubated with the membrane at room temperature for $2 \mathrm{~h}$. The primary antibodies used were as follows: Polyclonal primary rabbit anti-iNOS antibody (cat. no. SAB4502012, 
A

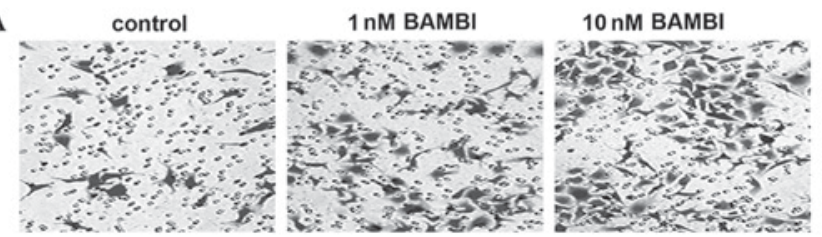

B

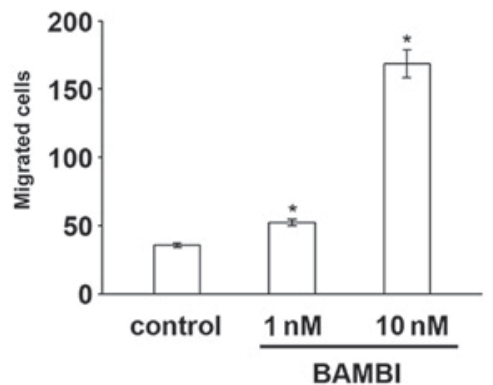

Figure 1. BAMBI induces the migration of monocytes. (A) Images of the migrated monocytes from a Boyden chamber assay. (B) The number of migrated cells in 10 visual fields at $\mathrm{x} 400$ magnification. ${ }^{*} \mathrm{P}<0.05$ vs. control. BAMBI, bone morphogenic protein and activin membrane-bound inhibitor homolog.

1:500 dilution; Sigma-Aldrich; Merck Millipore); polyclonal primary rabbit anti-interleukin (IL)-12 antibody (1:500 dilution, cat. no. SAB1306460; Sigma-Aldrich; Merck Millipore), polyclonal primary rabbit anti-IL-10 antibody (1:500 dilution, cat. no. SAB1410712; Sigma-Aldrich; Merck Millipore), polyclonal primary rabbit anti-arginase 1 antibody (1:500 dilution, cat. no. A6107; Sigma-Aldrich; Merck Millipore); and polyclonal primary rabbit anti-GAPDH antibody (1:2,000 dilution, cat. no. SAB2108266; Sigma-Aldrich; Merck Millipore). Membranes were subsequently incubated with secondary antibody (goat anti-rabbit IgG-peroxidase antibody, cat. no. A0545, 1:2,000 dilution; Sigma-Aldrich; Merck Millipore) at room temperature for $1 \mathrm{~h}$. The blots were detected by SignalBoost ${ }^{\mathrm{TM}}$ Immunoreaction Enhancer kit (EMD Millipore) and visualized using a Kodak 440 digital imager (Kodak, Rochester, NY, USA). The analysis was performed using Kodak Molecular Imaging software, version 4.5 (Kodak). The relative protein levels were calculated using Image-Pro Plus software, version 5.0 (Media Cybernetics, Inc.) comparing the grayscale of the blots.

Immunohistochemistry. Tissue sections $(6 \mu \mathrm{m})$ from representative paraffin blocks were de-paraffinized and rehydrated using graded ethanol and then blocked using $4 \%$ normal rabbit serum (Beyotime Institute of Biotechnology) at room temperature for $15 \mathrm{~min}$. Then, primary monoclonal mouse anti-human CD68 antibody (1:200 dilution, cat. no. ab201340; Abcam, Cambridge, MA, USA) and mouse monoclonal anti-human BAMBI with $37^{\circ} \mathrm{C}$ for $1 \mathrm{~h}$ (1:200 dilution, cat. no. ab57043; Abcam) were combined with the avidin-biotin blocking solution and ABC staining kit (Santa Cruz Biotechnology, Inc., Dallas, TX, USA), according to the manufacturer's instructions. The sections were processed in a 3,3'-diaminobenzidine $/ \mathrm{H}_{2} \mathrm{O}_{2}$ solution and stained using hematoxylin (Beyotime Institute of Biotechnology). The experiment with the reagents were used according to the manufacturer's instructions. Staining was performed at room temperature for $10 \mathrm{~min}$. The stained
A

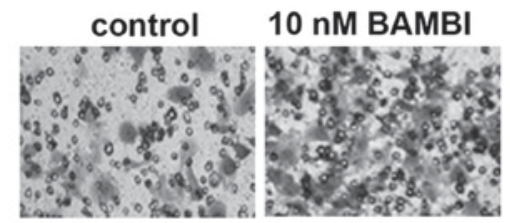

B

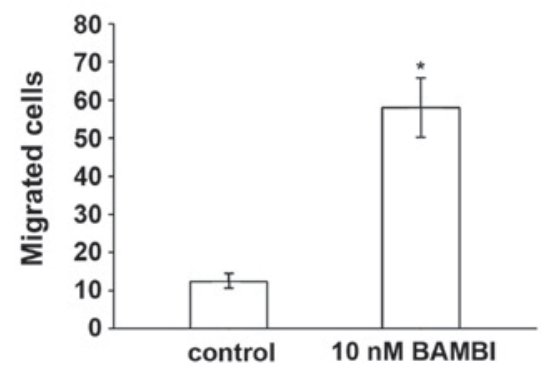

Figure 2. BAMBI promotes the migration of phorbol myristate acetate-induced macrophages. (A) Images of migrated monocytes from a Boyden chamber assay. (B) The number of migrated cells in 10 visual fields at $\mathrm{x} 400$ magnification. ${ }^{*} \mathrm{P}<0.05$. BAMBI, bone morphogenic protein and activin membrane-bound inhibitor homolog.

sections were observed and images captured using a Leica 400 light microscope (Leica Microsystems, Inc.).

Green-fluorescent immunostaining was performed using primary monoclonal mouse anti-human CD68 antibody (1:1,000 dilution, cat. no. ab201340; Abcam) and mouse monoclonal anti-human BAMBI $(1: 1,000)$ at $37^{\circ} \mathrm{C}$ for $1 \mathrm{~h}$. The secondary antibody was Goat Anti-Mouse IgG H\&L (FITC) (1:1,000, cat. no. ab6785; Abcam), which was incubated at room temperature for $15 \mathrm{~min}$. The stained cells were observed and images captured using fluorescence microscope (DMI 3000B; Leica Microsystems, Inc.).

Statistical analysis. Data are presented as the mean \pm standard deviation from three independent experiments, each performed in triplicate or quadruplicate. Statistical evaluation of data was performed using Student's t-test, one-way analysis of variance (ANOVA) or repeated measures ANOVA (SPSS 13.0 software; SPSS, Inc., Chicago, IL, USA). The Pearson correlation coefficient $(\mathrm{R})$ test was performed for correlation analysis. $\mathrm{P}<0.05$ was considered to indicate a statistically significant difference.

\section{Results}

BAMBI induces the migration of monocytes and macrophages in vitro. The present study assayed the effect of BAMBI on monocyte cells migration using Boyden chambers. RAW 264.7 monocytes were seeded in the top compartment of the chamber and enhancing concentrations of recombinant human BAMBI was added to the bottom compartment. The results demonstrated that BAMBI stimulated monocyte migration in a dose-dependent manner compared with the control cells $(\mathrm{P}=0.02$; Fig. 1). To identify the on chemically-differentiated monocytes, PMA was used to stimulate the monocytes to differentiate into macrophages. BAMBI also significantly increased macrophage migration compared with the control group ( $\mathrm{P}=0.03$; Fig. 2 ), providing further evidence of the effects of BAMBI on inflammatory cells. 
A

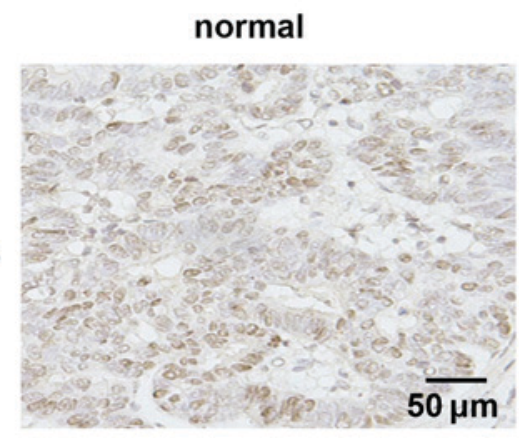

B

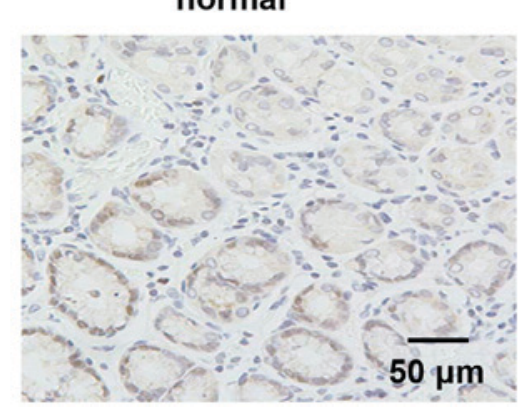

gliomas

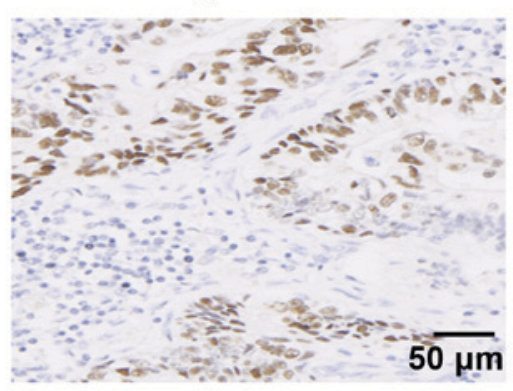

gliomas

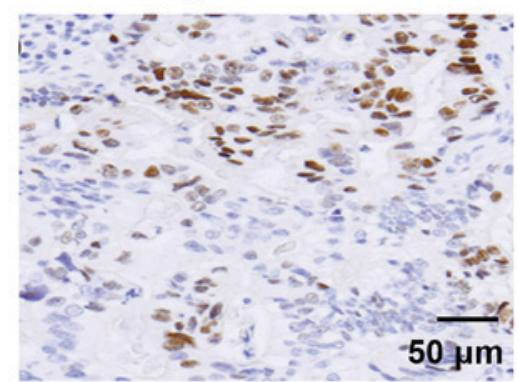

Figure 3. Immunohistochemical staining of normal tissue and glioma. (A) CD68 and (B) BAMBI expression in normal and gliomas tissues. BAMBI, bone morphogenic protein and activin membrane-bound inhibitor homolog.

A

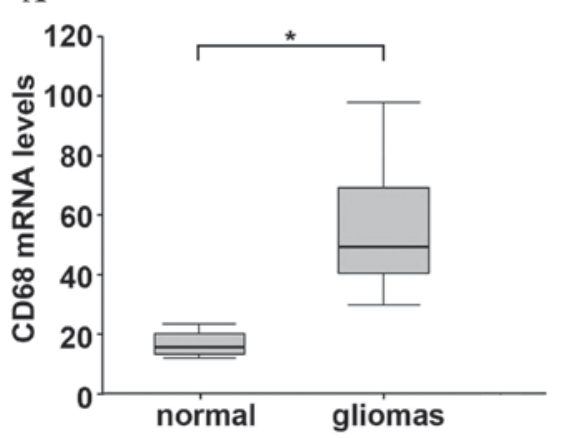

B

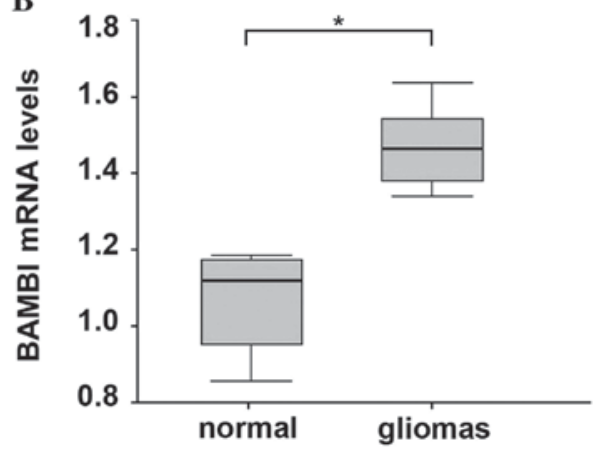

Figure 4. Quantitative polymerase chain reaction analysis of (A) CD68 and (B) BAMBI mRNA levels in normal and gliomas tissues. ${ }^{*} \mathrm{P}<0.05$ vs. control.

Positive correlation between BAMBI expression and macrophage levels. In order to validate the in vitro findings, analysis was performed on a retrospective selection of gliomas tissue specimens. CD68 is a macrophage-specific marker, and thus, was used to detect macrophages. High levels of CD68 were observed in glioma samples compared with the control specimens (Fig. 3A). Additionally, compared with normal specimens, high levels of BAMBI were detected in the gliomas tissues (Fig. 3B). The localization of BAMBI and number of macrophages exhibited similar patterns in normal and glioma tissues. qPCR analysis demonstrated that CD68 expression was significantly increased in glioma compared with normal tissue, which is similar to the result of immunohistochemistry analysis $(\mathrm{P}=0.01$; Fig. 4A). Additionally, the BAMBI mRNA was significantly increased in glioma samples compared with normal tissues ( $\mathrm{P}=0.02$; Fig. 4B). Furthermore, correlation analysis demonstrated that BAMBI is positively correlated with CD68 in gliomas $(\mathrm{P}<0.01$ and $\mathrm{R}^{2}=0.64$; Fig. 5).

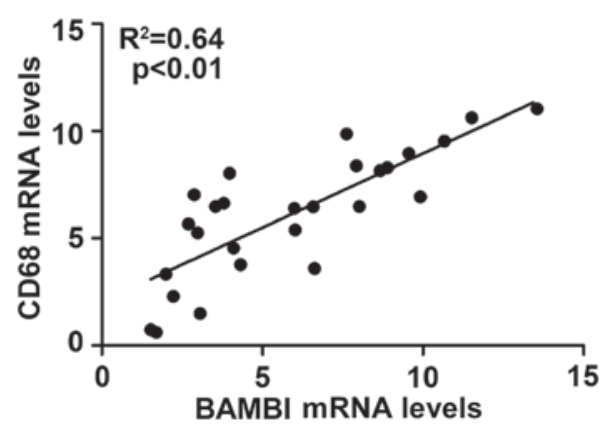

Figure 5. Correlation analysis of BAMBI and CD68 mRNA levels in 27 gliomas specimens. BAMBI, bone morphogenic protein and activin membrane-bound inhibitor homolog.

$B A M B I$ promotes the differentiation of macrophages. To determine whether BAMBI induces the proliferation of macrophages during tumorigenesis, the co-treatment of BAMBI and PMA was performed on RAW 264.7 macrophages (Fig. 6). 

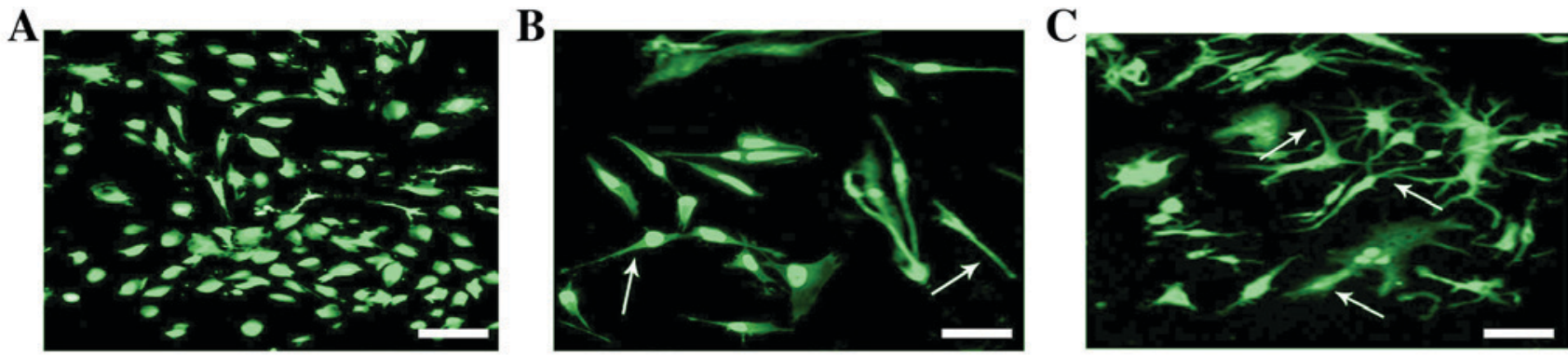

Figure 6. BAMBI-induced macrophage differentiation exhibited by green-fluorescent immunostaining. (A) RAW 264.7 treated with $0.1 \%$ fetal bovine serum for $48 \mathrm{~h}$ as control. (B) RAW 264.7 treated with $10 \mathrm{nM}$ BAMBI for $48 \mathrm{~h}$. (C) RAW 264.7 treated with $10 \mathrm{nM}$ BAMBI and $50 \mathrm{nM}$ phorbol myristate acetate for $48 \mathrm{~h}$. The arrows demonstrate the dendrite-like cells. Scale bar, $100 \mu \mathrm{m}$. BAMBI, bone morphogenic protein and activin membrane-bound inhibitor homolog.

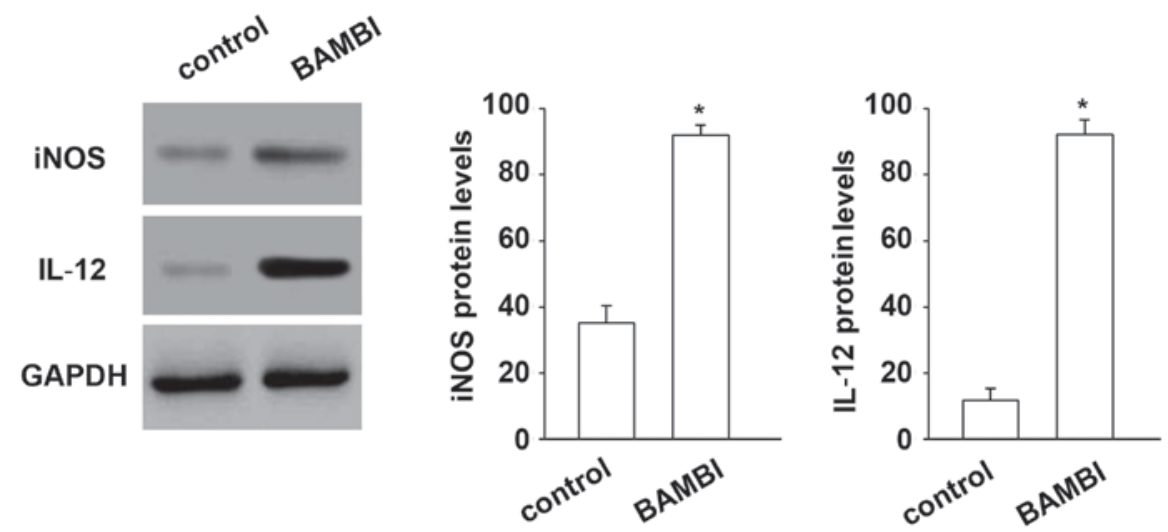

Figure 7. Western blot analysis indicates that BAMBI promotes the protein expression of iNOS and IL-12. "P $<0.05$ vs. control. $n=3$. BAMBI, bone morphogenic protein and activin membrane-bound inhibitor homolog; iNOS, inducible nitric oxide synthase; IL-12, interleukin-12.
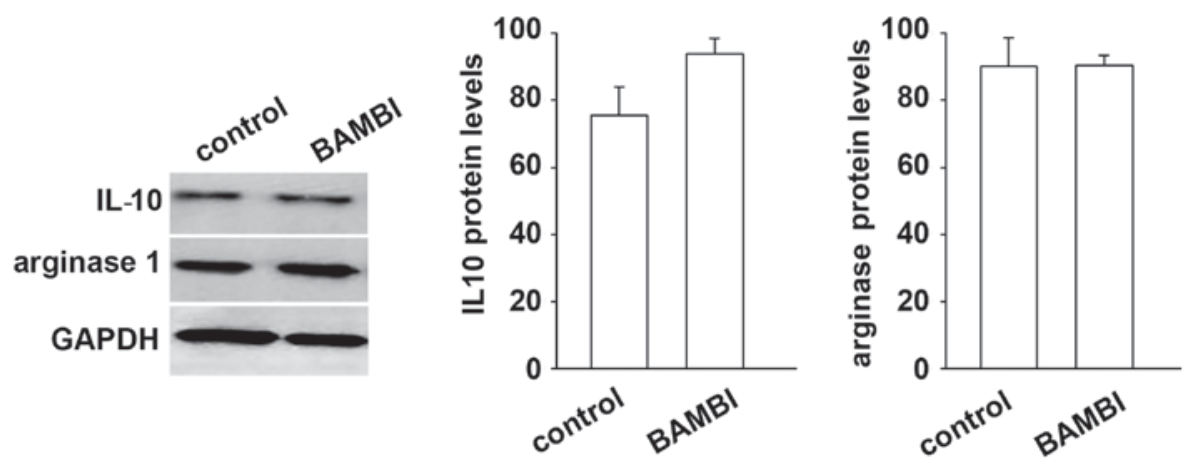

Figure 8. BAMBI exerts no effect on the expression of IL-10 and arginase 1, as demonstrated by western blot analysis. n=3. BAMBI, bone morphogenic protein and activin membrane-bound inhibitor homolog; IL-10, interleukin-10.

The result demonstrated that after $48 \mathrm{~h}$ treatment with BAMBI, certain cells were into dendrite-like. With BAMBI and PMA treatment, the number of dendrite-like cells was increased compared with BAMBI-only treatment. This indicated that BAMBI and PMA promote the differentiation of macrophages.

BAMBI induces differentiation of $M 1$ macrophages. In order to improve the description of the BAMBI-treated macrophage phenotype, the expression of specific M1 (iNOS and IL-12) and M2 (IL-10 and arginase 1) macrophages markers was performed on BAMBI-treated RAW 264.7 cells. The results demonstrated that the protein levels of specific markers of M1 macrophages (iNOS and IL-12) were increased significantly after by with BAMBI compared with the control, indicating that BAMBI induces differentiation of monocytes to M1 type macrophages (Fig. 7). By contrast, the markers of M2 type macrophages, including IL-10 and arginase 1 were not affected by BAMBI treatment (Fig. 8). These results indicated that BAMBI induced differentiation of M1 type macrophages.

\section{Discussion}

Gliomas consist of two key cellular compartments; the epithelium and the surrounding stroma. Glioma cell invasion is regulated by microregional extracellular matrix 
heterogeneity $(20,21)$. Among the inflammatory cells, macrophages have been reported to be involved in various processes associated with inflammation during tumorigenesis (22-24). However, the specific mechanisms involved and the prognostic importance of macrophages in tumorigenesis remains unclear; investigating this is important to verify additional markers. Higher expression of BAMBI in different types of cancer has been reported previously $(11,13)$ and has been suggested that BAMBI modulates inflammation (16). To the best of our knowledge, the present study is the first to demonstrate that BAMBI stimulates the migration of monocytes and macrophages in vitro. In addition, the expression levels of BAMBI were correlated with macrophage density (indicated by CD68 expression) in human gliomas specimens. It was also illustrated that BAMBI induced the expression of certain M1-specific markers, which emphasized the inflammatory-modulating effect in glioma.

BAMBI expression has been previously reported to be increased in colorectal (25) and ovarian (26) cancer. In the majority of these types of cancer, higher BAMBI expression indicated poor prognosis. A previous study suggested that BAMBI potentially regulated inflammation, particularly acting on monocytes and macrophages. The role of macrophages in cancer development is controversial. During cancer progression, polarized M2 macrophages are induced by tumor-derived and T cell-derived cytokines (9). The ratio of M2 macrophages is an indicator of poor prognosis (27). Additionally, tumor necrosis factor was demonstrated to inhibit tumor growth in the brain by promoting the recruitment of macrophages (28), which also indicated that macrophages have an anticancer effect via gene regulation.

The present study demonstrated that compared with normal tissues, macrophage density was increased in glioma samples $(17,23)$. A previous study reported that the total number of macrophages was positively correlated with recurrence-free survival following radical treatment (29). The contradiction between these finding may be due to the role of the two different macrophages phenotypes: M1-type (classically activated) and M2-type (alternatively activated) (30). M1-type macrophages generally express iNOS, which is tumor-cytotoxic. Tumor progression stimulates a phenotypic switch to M2 macrophages that express arginase 1 , and accelerate tumor growth, survival and metastasis (31). Similarly, it has been previously demonstrated that the density of iNOS-positive macrophages, which infiltrate the stroma, is decreased in highly aggressive gliomas compared with less aggressive cancer, which indicates that as malignant potential of the cancer increased, the cytotoxic activity of macrophages is reduced (32). The present study demonstrated that treatment with BAMBI increased the expression of M1-specific markers and had no effect on the expression of M2 markers, which suggests that BAMBI promoted differentiation of M1 macrophages. Thus, we hypothesize that higher expression of BAMBI may inhibit tumorigenesis.

The outcomes of the present study are valuable as they demonstrate the role of BAMBI in gliomas. BAMBI stimulated the migration of monocytes and promoted their differentiation toward the M1 inflammatory pathway. The results also indicate that macrophages and BAMBI may exert important function in glioma.

\section{References}

1. Komohara Y, Ohnishi K, Kuratsu J and Takeya M: Possible involvement of the M2 anti-inflammatory macrophage phenotype in growth of human gliomas. J Pathol 216: 15-24, 2008.

2. Abou-Ghazal M, Yang DS, Qiao W, Reina-Ortiz C, Wei J,Kong LY, Fuller GN, Hiraoka N, Priebe W, Sawaya R and Heimberger AB: The incidence, correlation with tumor-infiltrating inflammation and prognosis of phosphorylated STAT3 expression in human gliomas. Clin Cancer Res 14: 8228-8235, 2008.

3. Black KL, Chen K, Becker DP and Merrill JE: Inflammatory leukocytes associated with increased immunosuppression by glioblastoma. J Neurosurg 77: 120-126, 1992.

4. Ali S, King GD, Curtin JF, Candolfi M, Xiong W, Liu C, Puntel M, Cheng Q, Prieto J, Ribas A, et al: Combined immunostimulation and conditional cytotoxic gene therapy provide long-term survival in a large glioma model. Cancer Res 65: 7194-7204, 2005.

5. Dewey RA, Morrissey G, Cowsill CM, Stone D, Bolognani F, Dodd NJ, Southgate TD, Klatzmann D, Lassmann H, Castro MG and Löwenstein PR: Chronic brain inflammation and persistent herpes simplex virus 1 thymidine kinase expression in survivors of syngeneic glioma treated by adenovirus-mediated gene therapy: Implications for clinical trials. Nat Med 5: 1256-1263, 1999.

6. Nishie A, Ono M, Shono T, Fukushi J, Otsubo M, Onoue H, Ito Y, Inamura T, Ikezaki K, Fukui M, et al: Macrophage infiltration and heme oxygenase-1 expression correlate with angiogenesis in human gliomas. Clin Cancer Res 5: 1107-1113, 1999.

7. Balkwill $\mathrm{F}$ and Mantovani A: Inflammation and cancer: Back to Virchow? Lancet 357: 539-545, 2001.

8. Rosenman SJ, Shrikant P, Dubb L, Benveniste EN and Ransohoff RM: Cytokine-induced expression of vascular cell adhesion molecule-1 (VCAM-1) by astrocytes and astrocytoma cell lines. J Immunol 154: 1888-1899, 1995.

9. Mantovani A, Sozzani S, Locati M, Allavena P and Sica A: Macrophage polarization: Tumor-associated macrophages as a paradigm for polarized M2 mononuclear phagocytes. Trends Immunol 23: 549-555, 2002.

10. Gabrusiewicz K, Ellert-Miklaszewska A, Lipko M, Sielska M, Frankowska M and Kaminska B: Characteristics of the alternative phenotype of microglia/macrophages and its modulation in experimental gliomas. PLoS One 6: e23902, 2011.

11. Sekiya T, Adachi S, Kohu K, Yamada T, Higuchi O, Furukawa Y, Nakamura Y, Nakamura T, Tashiro K, Kuhara S, et al: Identification of BMP and activin membrane-bound inhibitor (BAMBI), an inhibitor of transforming growth factor-beta signaling, as a target of the beta-catenin pathway in colorectal tumor cells. J Biol Chem 279: 6840-6846, 2004.

12. Grotewold L, Plum M, Dildrop R, Peters T and Rüther U: Bambi is coexpressed with Bmp-4 during mouse embryogenesis. Mech Dev 100: 327-330, 2001

13. Blanco Calvo M, Bolós Fernández V, Medina Villaamil V, Aparicio Gallego G, Díaz Prado S and Grande Pulido E: Biology of BMP signalling and cancer. Clin Transl Oncol 11: 126-137, 2009.

14. Sekiya T, Oda T, Matsuura K and Akiyama T: Transcriptional regulation of the TGF-beta pseudoreceptor BAMBI by TGF-beta signaling. Biochem Biophys Res Commun 320: 680-684, 2004.

15. Onichtchouk D, Chen YG, Dosch R, Gawantka V, Delius H, Massagué $\mathbf{J}$ and Niehrs C: Silencing of TGF-beta signalling by the pseudoreceptor BAMBI. Nature 401: 480-485, 1999.

16. Xavier S, Gilbert V, Rastaldi MP, Krick S, Kollins D, Reddy A, Bottinger E, Cohen CD and Schlondorff D: BAMBI is expressed in endothelial cells and is regulated by lysosomal/autolysosomal degradation. PLoS One 5: e12995, 2010.

17. Eccles SA: Macrophages and cancer. In: Immunological Aspects of Cancer. Springer, pp123-154, 1978.

18. Marwitz S, Droemann D, Rupp J, Rohmann K, Osbahr S, Ulmer AJ, Röschmann K, Abdullah M, Schultz H, Vollmer E, et al: BAMBI-A TGF- $\beta$ pseudoreceptor with possible functional involvement in COPD and NTHI infection. Eur Respir J 38: 4752, 2011.

19. Livak KJ and Schmittgen TD: Analysis of relative gene expression data using real-time quantitative PCR and the 2(-Delta Delta C(T)) method. Methods 25: 402-408, 2001.

20. Bellail AC, Hunter SB, Brat DJ, Tan C and Van Meir EG: Microregional extracellular matrix heterogeneity in brain modulates glioma cell invasion. Int J Biochem Cell Biol 36: 1046-1069, 2004. 
21. Rutka JT, Apodaca G, Stern R and Rosenblum M: The extracellular matrix of the central and peripheral nervous systems: Structure and function. J Neurosurg 69: 155-170, 1988.

22. Pollard JW: Tumour-educated macrophages promote tumour progression and metastasis. Nat Rev Cancer 4: 71-78, 2004.

23. Condeelis J and Pollard JW: Macrophages: Obligate partners for tumor cell migration, invasion, and metastasis. Cell 124: 263-266, 2006.

24. Bach JP, Deuster O, Balzer-Geldsetzer M, Meyer B, Dodel R and Bacher M: The role of macrophage inhibitory factor in tumorigenesis and central nervous system tumors. Cancer 115: 2031-2040, 2009.

25. Fritzmann J, Morkel M, Besser D, Budczies J, Kosel F, Brembeck FH, Stein U, Fichtner I, Schlag PM and Birchmeier W: A colorectal cancer expression profile that includes transforming growth factor beta inhibitor BAMBI predicts metastatic potential. Gastroenterology 137: 165-175, 2009.

26. Pils D, Wittinger M, Petz M, Gugerell A, Gregor W, Alfanz A, Horvat R, Braicu EI, Sehouli J, Zeillinger R, et al: BAMBI is overexpressed in ovarian cancer and co-translocates with Smads into the nucleus upon TGF-beta treatment. Gynecol Oncol 117: $189-197,2010$

27. Niino D, Komohara Y, Murayama T, Aoki R, Kimura Y, Hashikawa K, Kiyasu J, Takeuchi M, Suefuji N, Sugita Y, et al: Ratio of M2 macrophage expression is closely associated with poor prognosis for angioimmunoblastic T-cell lymphoma (AITL). Pathol Int 60: 278-283, 2010.
28. Villeneuve J, Tremblay P and Vallières L: Tumor necrosis factor reduces brain tumor growth by enhancing macrophage recruitment and microcyst formation. Cancer Res 65: 3928-3936, 2005.

29. Shimura S, Yang G, Ebara S, Wheeler TM, Frolov A and Thompson TC: Reduced infiltration of tumor-associated macrophages in human prostate cancer: Association with cancer progression. Cancer Res 60: 5857-5861, 2000.

30. Umemura N, Saio M, Suwa T, Kitoh Y, Bai J, Nonaka K, Ouyang GF, Okada M,Balazs M,Adany R, et al: Tumor-infiltrating myeloid-derived suppressor cells are pleiotropic-inflamed monocytes/macrophages that bear M1- and M2-type characteristics. J Leukoc Biol 83: 1136-1144, 2008.

31. Nelius T, Samathanam C, Martinez-Marin D, Gaines N, Stevens J, Hickson J, de Riese W and Filleur S: Positive correlation between PEDF expression levels and macrophage density in the human prostate. Prostate 73: 549-561, 2013.

32. Tsai CS, Chen FH, Wang CC, Huang HL, Jung SM, Wu CJ, Lee CC, McBride WH, Chiang CS and Hong JH: Macrophages from irradiated tumors express higher levels of iNOS, arginase-I and COX-2, and promote tumor growth. Int J Radiat Oncol Biol Phys 68: 499-507, 2007. 